\title{
Optimized sequential method assisting design and implementation of electro- pneumatic/hydraulic circuit
}

\author{
Vinicius Abrão S. Marques, Felipe Nascimento C. Rodrigues, José Jean-Paul Z. de S. Tavares.
}

\begin{abstract}
Electro-pneumatic circuits returned to be an important topic of research after the need of ventilator due COVID-19. Not only for this type of device but to assist economy restarting, the electropneumatic/hydraulic systems will be one of the industrial automation cornerstones after COVID pandemic. Programmable Logic Controllers (PLC) are one of the main components of these circuits and the standard of industrial automation; however, its application is highly dependent on programmers skills. Usually, electro-pneumatic/hydraulic solutions rely on sequential circuits. Different methods are found in the literature to design that systems, but they involve complex analyzes and intuitive strategies in cases that the actuators extend and retract more than once within the cycle of movements, and within simultaneous movements. As consequence, the resulting circuits mostly require a large number of valves and electronic components in order to be implemented. The objective of this work is to describe a novel method that can be applied in the design and implementation of optimized circuits using PLC's, eliminating intuitive solutions. Examples are presented to illustrate the theoretical procedures and experimental tests performed to validate the method. The results prove the generality of this work, the simplicity of its implementation, and introduce a bridge between design and implementation of electropneumatics and electro/hydraulics circuits with PLC's.
\end{abstract}

Keywords - Electro-Pneumatic/Hydraulic Systems, Sequential Logic Circuits, Programmable Logic Controller.

\section{INTRODUCTION}

$\mathrm{E}$ lectro-pneumatic systems are back in evidence due the emergency design of ventilators for critically ill patients with COVID-19 (MIT,2020)(OSV,2020). Industrial sequential electro-pneumatic/hydraulic circuits are those in which the actuators move in accordance with a model that repeats over time. It is utilized by various industrial applications to solve problems of production automation, with relative low cost. In the currently global scenario this kind of solution will be mandatory to rebuild

Vinicius Abrão S. Marques is with the Department of Mechanical Engineering, Federal University of Triângulo Mineiro, Brazil. (Phone: +55-34-988434305, e-mail: vinicius.marques@uftm.edu.br).

José Jean-Paul Z. de S. Tavares is with the Faculty of Mechanical Engineering, Federal University of Uberlândia, Brazil. (email: jean.tavares@ufu.br)

Felipe Nascimento Cerqueira Rodrigues was with the Department of Mechanical Engineering, Federal University of Triângulo Mineiro, Brazil. (Phone: +55-34-999949720, e-mail: felipenascimento028@gmail.com). human economy after the pandemic lockdown.

According to Bayoumi (2014) and Rohner (1979), there is no method that can be applied to all sequential circuits, highlighting some of the most used as Karnaugh maps, step counter, combinational logic circuit design and cascading method (Malayappan et. al., 2009). Cheng (1973) describes a simple time-chart method of analysis for pneumatics circuits. Novais (1997) proposes a sequential method for the solution of electro-pneumatic circuits, but since then, the memory valves have been replaced by electrical relays which allow optimizing the circuits reducing the number of components used. Bonacorso e Noll (1997) also discusses the sequential method proposed by Novais (1997), using relays and applying to problems in which the actuators advance and return more than once during the cycle of movement, but limited to non-complex systems without guarantee of functional solution. Another method called path-step (Bollmann, 1996) solves the circuit intuitively. A comparison among methods of multi actuators circuits design is described in Jagadeesha (2014). An evolutionary algorithm for programming flexible pneumatics circuits was proposed by Ganesh and Gurunathan (2011) (2017), however, despite the fact it presents the solution automatically for PLC without designer's validation, it is not user-friendly to be applied in shop-floor application level. In respect of solving sequential circuits, those methods involve complex analyzes and result, most of time, in circuits that require a large number of components in order to implement the solutions obtained.

This paper describes an electro-pneumatic/hydraulic sequential method which is able to optimize the circuits, eliminating intuitive solutions, taking account hardware restrictions (number of available types of valve, relays and additional gadgets) and user friendly in shop floor level. Besides these originalities, the proposed method can be applied in the project of circuits with sequences of simultaneous movements of actuators and with repetition, defining a procedure to design the electric seals, using or not PLCs. Examples of theoretical procedures are provided and experimental tests were done to validate the methodology.

Section II presents the proposed method for the design of sequential circuits and a comparison with other methods from the literature. Section III shows the design and implementation of a sequential circuit that includes repetition of movement and simultaneous movements of the actuators. Section IV highlights the final considerations, followed by the references. 


\section{II.OPTIMIZED SEQUENTIAL METHOD}

In this work the following nomenclature is considered in the definition of the proposed method:

$i \mathrm{~S} 1$ : Sensor detects that actuator $i$ is retracted, for $i \in \mathcal{N}$ ( $\mathrm{i}=1$ for actuator $A, \mathrm{i}=2$ for actuator $B$, and so on);

$i \mathrm{~S} 2$ : Sensor detects that actuator $i$ is extended;

$i$ Y1: Solenoid that controls the extension of actuator $i$;

$i$ Y 2 : Solenoid that controls the retraction of actuator $i$;

$A+$ : Extension movement of actuator $A$;

$A$-: Retraction movement of actuator $A$.

The nomenclature is illustrated in Fig. 1.

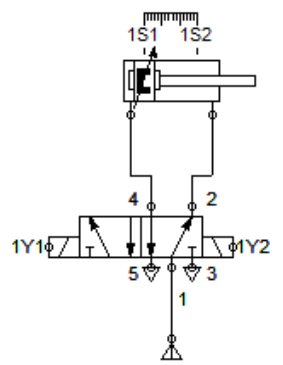

Fig. 1. Exemplification of the nomenclature.

The steps of the proposed method can be divided in 8 (eight) parts as following.

2.1 Division of groups based on cascade method (Bonacorso and Noll,1997)

Step 1 - To identify whether the sequence is direct or indirect: The procedure starts by written the sequence in the abbreviated form.

Examples:

$\mathrm{A}+\mathrm{A}-\mathrm{B}+\mathrm{B}-$

$\mathrm{A}+\mathrm{B}+\mathrm{B}-\mathrm{A}-$

$\mathrm{A}+\mathrm{C}+\mathrm{B}-\mathrm{A}-\mathrm{C}-\mathrm{B}+$.

Then, a vertical line must be passed, dividing the sequence exactly in the middle:

$\mathrm{A}+\mathrm{B}+\mid \mathrm{A}-\mathrm{B}-$

$\mathrm{A}+\mathrm{A}-\mid \mathrm{B}+\mathrm{B}-$

$\mathrm{A}+\mathrm{C}+\mathrm{B}-\mid \mathrm{A}-\mathrm{C}-\mathrm{B}+$.

If the two sides of the trace are equal, it means, if they have the same letters and in the same order, it is a direct sequence, whose control circuit can be obtained without overlapping of signals. Otherwise, it is an indirect sequence. For instance:

$\mathrm{A}+\mathrm{B}+\mid \mathrm{A}-\mathrm{B}$ - results in

$\mathrm{A} B \mid \mathrm{A} B$. In this way, the sequence is direct.

$\mathrm{A}+\mathrm{A}-\mid \mathrm{B}+\mathrm{B}-$ results in

A A | B B. In this way, the sequence is indirect.

Another situation that characterizes an indirect sequence is when a letter appears more than once on one side of the dash, which generally indicates that an actuator performs two or more forward and reverse movements in a command cycle. As example:

$$
\mathrm{A}+\mathrm{B}+\mathrm{A}-\mathrm{A}+\mathrm{B}-\mathrm{A}+
$$

$\mathrm{A}+\mathrm{B}+\mathrm{B}-\mathrm{A}-\mathrm{B}+\mathrm{B}-$

are indirect sequence commands.

In case of direct sequence it is not required the use of relays, then the method follows to the subsection 2.2.

Step 2- Division in secondary sectors: Only in case of indirect sequences, the next step is to divide the sequence into secondary sectors that determine the size of the electric circuit and the number of relays to be used. To divide a sequence into sectors, it is first necessary to write the sequence again in abbreviated form. Then, the sequence should be read from left to right, cutting with a new vertical trace every time a letter is repeated, regardless of the forward (+) or indent (-) signs. Finally, the number of subdivision caused by these vertical traces, is equal to the number of secondary sectors that the electric circuit must have. Following an example:

$\begin{array}{lll}\mathrm{A}+\mid & \mathrm{A}-\mathrm{B}+\mid & \mathrm{B}- \\ \mathrm{I} & \text { II } & \text { I. }\end{array}$

Although, in this example, the traces have fractioned the sequence into three parts, if the letter contained in the last division is not contained in the first, the sectors can be unified with the same identification (in this case I), since the movement is cyclic. Thus, in order to optimize the number of relays, one can consider the return of B as an integral part of the first sector. Therefore, it requires 2 secondary sectors for the electric circuit. Other examples of 2 and 4 sectors, respectively, are:

$\mathrm{A}+\mathrm{B}+\mathrm{B}-\mathrm{A}-$

I II

$\begin{array}{llll}\mathrm{A}+\mathrm{B}+\mid & \mathrm{B}-\mathrm{A}-\mid & \mathrm{B}+\mid & \mathrm{B}- \\ \mathrm{I} & \text { II } & \text { III } & \text { IV }\end{array}$

Unlike the sequence $\mathrm{A}+|\mathrm{A}-\mathrm{B}|+\mathrm{B}-$, where the last division was considered an integral part of the first one, since the letters did not repeat themselves, it cannot be used the same strategy in the sequence $\mathrm{A}+\mathrm{B}+|\mathrm{B}-\mathrm{A}-| \mathrm{B}+\mid$ $\mathrm{B}-$, because the letter $\mathrm{B}$ that appears in the last division is also present in the first. In this way one should consider the rule in which a letter must be present only once in each subdivision.

Step 3: Design the electric cascade: The next step is to design the electrical circuit according to the number of secondary sectors found in previous step. The number of auxiliary relays that should control the circuit, energizing one sector at a time and avoiding overlapping signals, is given by (1).

$$
n_{r}=n_{s}-1
$$

Where $n_{r}$ is the number of relays and $n_{s}$ is the number of sectors.

Fig. 2 and Fig. 3 represent the electrical circuits for different numbers of secondary sectors.

2.2 - Construction of path-step diagrams similar to Bollmann (1996): 


$$
\text { K1 } \oint_{2}^{1} \mathrm{~K}_{1} \oint_{4}^{3}
$$

Fig. 2. Electrical circuit with 1 relay for 2 sectors.

$$
\text { K2 }
$$$$
\text { । } 11 \quad 111
$$

Fig. 3. Electrical circuit with 2 relays for 3 sectors.

In a project of sequential circuits, using the optimized sequential method, it is necessary to represent the sequence of the actuators movement through the path-step diagram.

In the diagram of actuators their movements are represented by horizontal and equidistant lines. The movements are designed without vertical scale and it is the same for all actuators. Each actuator can assume only two positions, 0 or 1 , which means that the actuator is retracted or extended, respectively.

The sequence that is used to exemplify the optimized sequential method is $\mathrm{A}+\mathrm{B}+\mathrm{B}-\mathrm{A}-$. Fig. 4 shows the diagram of actuators for this sequence.

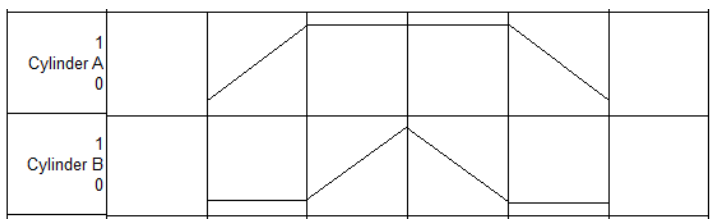

Fig. 4. Diagram of actuators of the sequence A + B + B- A-.

The next step is the construction of the correspondent diagram of sensors. In the diagram of sensors, the start and end of each sensor signal are represented by a closed circle on the corresponding step, and the duration of the signal is represented by a horizontal continuous line during the steps in which the sensor is enabled. Fig. 5 shows the diagram of sensors.

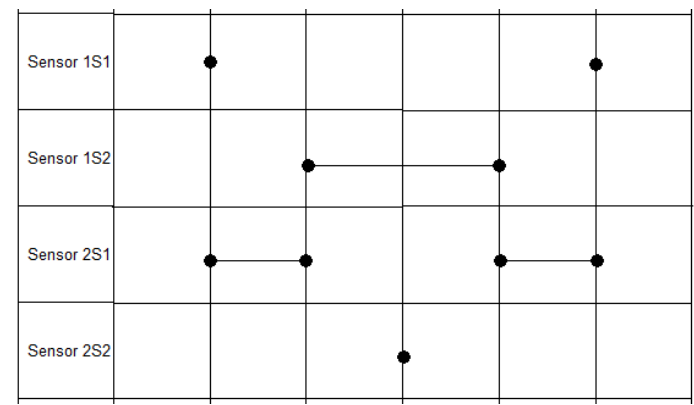

Fig. 5. Diagram of sensors of the sequence A + B + B- A-.

The next step is to obtain the diagram of actuators control. In the diagram, the beginning of the actuator command is represented by a closed circle and the end of the command by an open circle, on the corresponding steps, and the duration of the command is represented by a continuous horizontal line. The duration of the extension $(+)$ refers to the steps in which the actuator is advancing or is already extended. Similarly, the retraction (-) refers to the steps in which the actuator is retracting or is already retracted. Fig. 6 shows the diagram of actuators control.

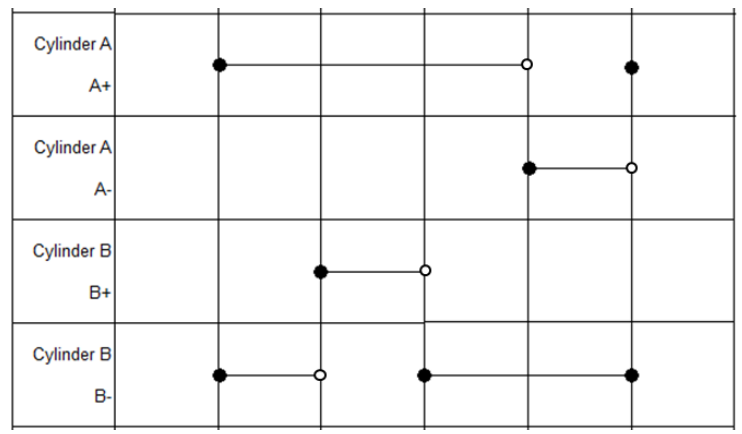

Fig. 6. Diagram of actuators control of the sequence $A+B+B-A-$.

When the sequence is indirect, it is necessary to use relays to define each sector, as defined in step 3 of subsection 2.1. Similarly, the diagram of the relays is obtained, as shown in Fig. 7.

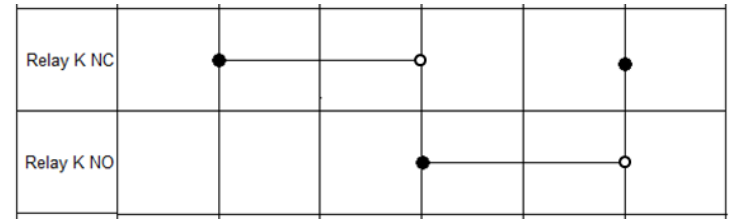

Fig. 7. Diagram of relays of the sequence A + B + B- A-

\subsection{Diagram Analysis}

In the design of the electro-pneumatic or hydraulic circuit it is initially defined what kind of directional valves should be used. There are two types of valves. On one hand, the bidirectional is a valve that has two actuating solenoids. On the other hand, the one direction or spring returning valve has only one solenoid and the actuator returns to the starting position by the action of a spring. According to the next steps of analysis of diagrams, simplifications can occur, converting, where possible, the command from bidirectional to one direction, due low cost; although it depends on the sequence of the movement and/or how much budget is available.

For the analysis of the diagrams one must consider the following three criteria:

1) One must use the logics AND (represented by *); OR (represented by + ); or both; to correlate the diagrams of sensors and relays, according to Fig. 8.

2) It is necessary to compare the start point of the actuator control signal with the start of the sensors and/or relays correlation, in order to find solutions that start together. 
3) Then, the duration of signals of the sensors and/or relays correlation, observed in the path-step diagram of the sensors and/or relays correlation, must be compared with the duration of the actuator command signals, as shown in the diagram of actuator control. If the durations are equal, an one direction valve can be applied; otherwise a bidirectional.

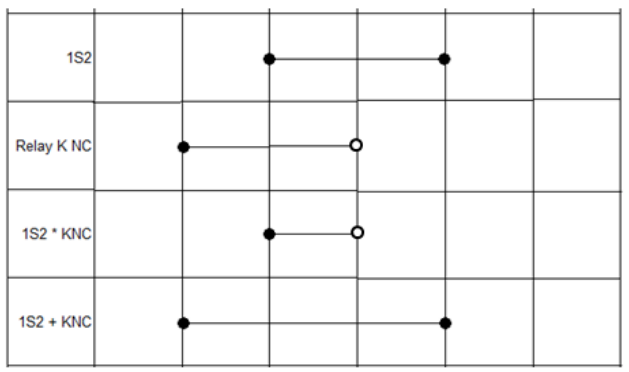

Fig. 8. Correlation of the sensor and relay diagrams.

Possible comparisons that meet criteria 2 and 3 are described by (2) for each of actuator command:

$\mathrm{A}+: 1 \mathrm{~S} 1 * \mathrm{KNC}$

A- : $2 \mathrm{~S} 1 * \mathrm{KNO}$

$\mathrm{B}+: 1 \mathrm{~S} 2 * \mathrm{KNC}$

B- : $2 \mathrm{~S} 2 \cdot{ }^{*} \mathrm{KNO}$

In (2), for each command evaluated, the sensor triggered at the previous movement from the sequence $\mathrm{A}+\mathrm{B}+\mathrm{B}-\mathrm{A}-\mathrm{is}$ associated with the relay of the sector in which the evaluated movement occurs. It should be noted, however, that this association is not mandatory, and the comparison of the actuator controls can be made with sensors AND/OR relays, only sensors or only relays.

Besides that, according to the criteria, the duration of the actuator control signal can never be smaller than sensors/relays signals. If it occurs, one must find another sensors and/or relays correlation. Fig. 9, Fig. 10 and Fig. 11 show possible associations.

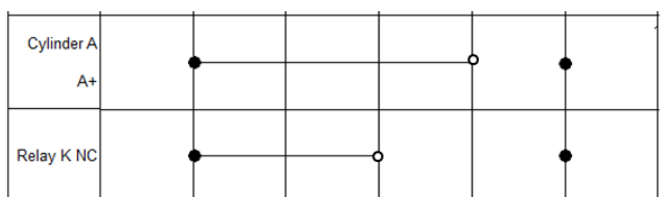

Fig. 9. The duration of the actuator command is greater than the relay

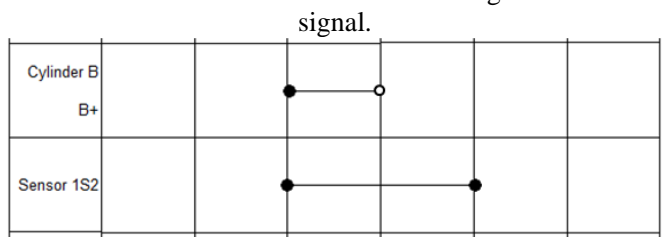

Fig. 10. The duration of the actuator command is shorter than the sensor

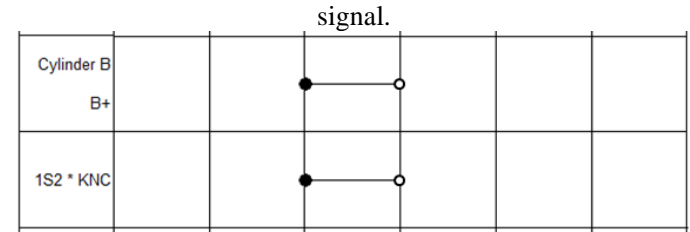

Fig. 11. The duration of the actuator control is equal to the relay signal associated with the sensor (AND logic).
Three possible scenarios can be found, and the solutions in each one are presented as follows:

i) If the duration of the + actuation is the same as the sensor and/or relay correlated, as illustrated by Fig. 11, this leads to a valve spring returned, as Fig. 12.

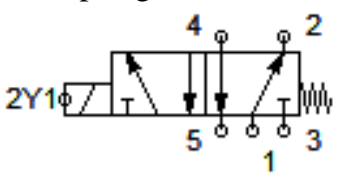

Fig. 12. Spring returned valve.

The use of a spring returned valve is justified since while an electric current is flowing through the solenoid $2 \mathrm{Y} 1$, the actuator keeps extending / extended, that is, both events have the same duration. As soon as the solenoid activation is stopped, the spring causes the actuator to return to the starting position.

ii) If the duration of + the actuation is longer than the sensor and/or relay correlated, the valve return control must be evaluated as follow.

Case the duration of the - actuation is the same as the sensor and/or relay correlated, as shown in Fig. 13, the bidirectional solenoid valve can be replaced by a one directional valve, as showed in Fig. 14.

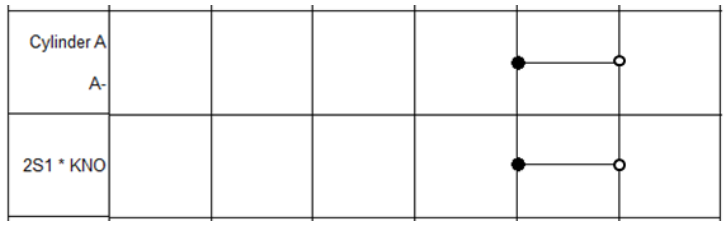

Fig. 13. 2S1 And KNO have the same duration as command A-.

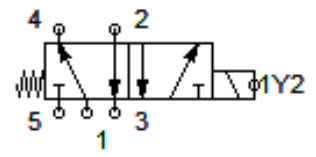

Fig. 14. One directional valve with advance control by spring.

In this case, we can invert the command to a valve spring returned by applying the logic presented in (3).

$$
A+=\overline{A-}=\overline{2 S 1 * K N O}=\overline{2 S 1}+K N C=2 S 1 N C+K N C
$$

That results in Fig. 15.

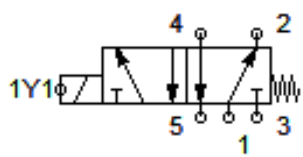

Fig. 15. Solenoid advance control and spring returned valve.

iii) If the actuator's advance and return commands duration are greater than the sensor and/or relay correlated, it is concluded that simplifications cannot be made, then a bi-directional solenoid valve must be used. Fig. 16 shows the bi directional valve representation. 


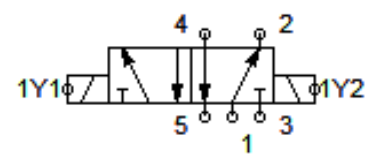

Fig. 16. Bi directional solenoid valve.

\subsection{Electric circuit}

Bonacorso e Noll (1997) presents some different choices of electrical sectors from Subsection 2.1, which may influence in the required amount of relays and bi directional valves instead of spring returned. In addition, different electrical sectors can even make it difficult to analyze the diagrams. Therefore, it is suggested to use the sector proposal presented in Subsection 2.1 which results, in general, in a large number of simplifications and optimizations (types of valve, number of relays and additional gadgets), as following illustrated.

From the results obtained in subsection 2.3 , it is possible to design the electric circuit. As shown, both actuators $A$ and $B$ can be simplified to work with spring returned valves. Then, the electric circuit of $A+(1 Y 1)$ and $\mathrm{B}+(2 \mathrm{Y} 1)$ is design according to the relationships obtained in (2) for $\mathrm{B}+$ and (3) for $\mathrm{A}+$, as shown in Fig. 17.

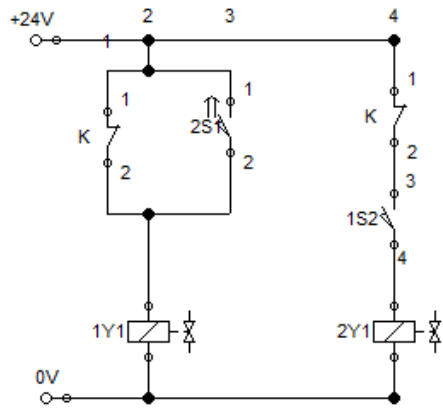

Fig. 17. Electric circuit of actuators for $\mathrm{A}+\mathrm{B}+\mathrm{B}-\mathrm{A}-$

It is observed in Fig. 17 that the sensor and relay connections are made in parallel, if associated by logic $\mathrm{OR}$, and in series, if associated by logic AND. The relay contacts are normally open, if associated with $\mathrm{KNO}$, or closed, case associated with KNC. Similarly, the contact of the sensors is drawn normally open, if it is directly associated with the sensor, or normally closed in case of associated with the barred sensor representation (Eq. (3)).

\subsection{Circuit of relay}

The electric circuit of relay seal is design as presented in Fig. 18. The ON and OFF contacts of the seal circuit are obtained according to the division of sectors, e.g., if the sequence is:

$\mathrm{A}+\mathrm{B}+\mid \mathrm{B}-\mathrm{A}-$,

$\mathrm{KNC} \quad \mathrm{KNO}$

the transition from $\mathrm{KNC}$ to $\mathrm{KNO}$ is defined by the movement of $\mathrm{B}+$, which occurs therefore when the sensor 2S2 is triggered, thus the ON contact of the seal is given by $2 \mathrm{~S} 2$. When the seal is turned on and the relay is energized, the sectors that have the KNO contact are energized.

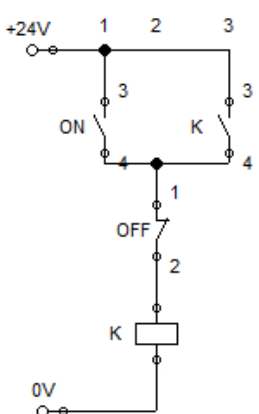

Fig. 18. Relay (ON and OFF) seal.

Similarly, the transition from KNO to KNC is defined by $\mathrm{A}-$, therefore $1 \mathrm{~S} 1$, thus the OFF contact is given by (1S1NC) that deactivates the relay and energizes the sectors that have the contact KNC.

Therefore, when the relay is activated, the second sector is energized and when the relay is deactivated, the first sector is energized. Fig. 19 shows the relay seal circuit.

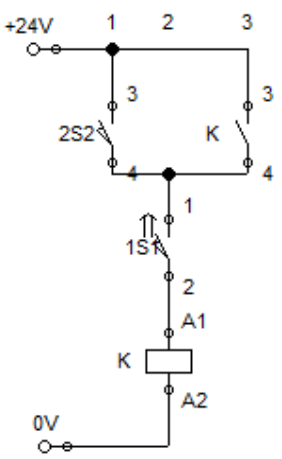

Fig. 19. Relay seal electric circuit for A+B+B-A-

It is observed in Fig. 19 that although the OFF contact is $1 \mathrm{~S} 1 \mathrm{NC}$, in the circuit the contact was drawn open, since in the initial condition the sensor $1 \mathrm{~S} 1$ is activated, causing the NC contacts to open.

Particular cases of seal design and choice of ON/OFF contacts occur when the same component is responsible for turning the seal $\mathrm{ON}$ and OFF or in cases where a component is triggered more than once during the motion sequence, which means there are repeated movements. It is, therefore, necessary to define a general methodology, valid even for such particular cases. Intuitive methods can be applied in these cases; however, the following steps can be applied regardless of the motion sequence:

I) Draw the diagrams according to subsection 2.2, including the diagram of the component responsible for turning the seal OFF. In this case, the diagram to be drawn is the component with normally closed contact (NC).

II) Then, the seal shutdown is evaluated: In this step, the $\mathrm{KNC}$ diagram is compared with the diagram of the 
NC component of previous item (I), according to Fig. 20.

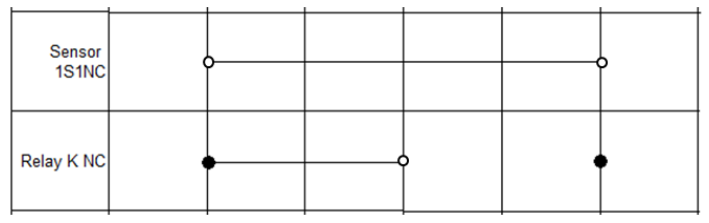

Fig. 20. Seal shutdown evaluation.

It is observed in Fig. 20 that the open circles of the diagram of the NC component must coincide with the signal of KNC. The reason is because the instant when the sensor NC is enabled (open circles in the diagram) must not coincide with the KNO signal. If it coincides the seal will shut down at an unexpected moment. In these cases, a second NO or NC contact of another component (sensor or even another relay) must be associated in parallel with the OFF contact of the sensor (NC contact). The resulting diagram of the association is again compared to the KNC diagram, seeking an association that results in all open circles coincident with KNC.

III) Evaluate the seal activation: In this step, the KNO diagram is compared with the diagram of component used in the ON contact associated (AND logic) with the solution of item (ii). It means that the activation of the seal depends on both the ON contact and the association responsible for the OFF (dominant shutdown seal). The diagram of this AND association is presented in Fig. 21.

As shown in Fig. 21, a solution is found if the result signal of the AND association starts simultaneously with the KNO and it has less or equal duration. If the duration is smaller, the seal itself keeps the relay energized. If the duration is greater, a normally open or closed contact of another sensor or relay should be associated in series with the $\mathrm{ON}$ contact in order to result in a final association that starts simultaneously with KNO and has a duration smaller than or equal to that of KNO.

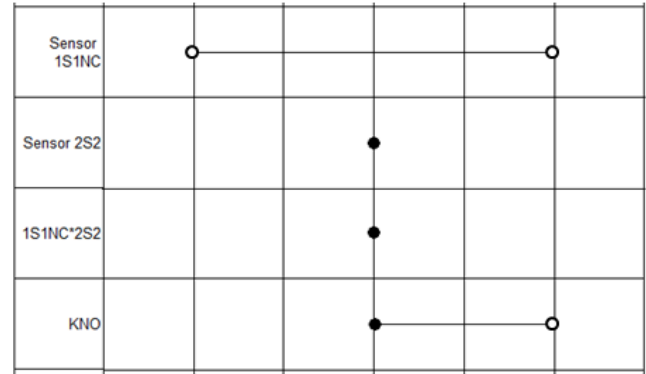

Fig. 21. Example of an AND association diagram starting with KNO.

\subsection{Start Button}

It is common in electro-pneumatic/hydraulic applications to implement a start button, which is responsible for initializing the sequence of the movements, as well as, when it is turned OFF, to cause the system to return to its initial condition.

According to the optimized sequential method, a start button can be added to the electrical circuit by associating one of its contacts normally open in series with the solenoid of the valve in its first movement, that may be in extend or retract movement (if the actuator starts extended). This association is justified since, when the button is activated, the normally open contact of button closes, allowing the passage of current at the instant that the solenoid activation logic is established. In addition, the button acts as an emergency key, energizing the system only when triggered.

If the valve is spring returned, when the button is turned OFF, the actuator returns immediately to its initial condition by the action of the spring. If the valve has double solenoid, it is still necessary to associate one of the normally closed contacts of the start button in parallel to the circuit of the solenoid that commands the return movement to the initial condition. When the button is turned OFF, it allows the passage of electric current directly to the solenoid moving the actuator back to initial position.

\subsection{Resulting circuit}

Both dual action actuators with $5 / 2$ or $4 / 2$ valves (see Fig.22 and 23) and single acting actuators with 3/2-way valves (see Fig.23) can be used in electro-pneumatic systems and 3-position valves in the electro hydraulic system.

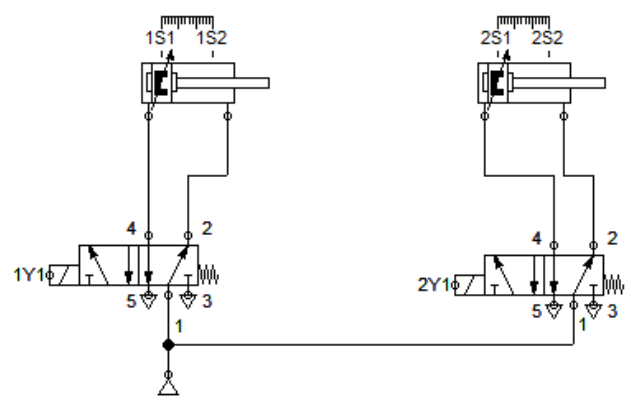

Fig. 22. Possible choice of actuators using 5/2 valves.

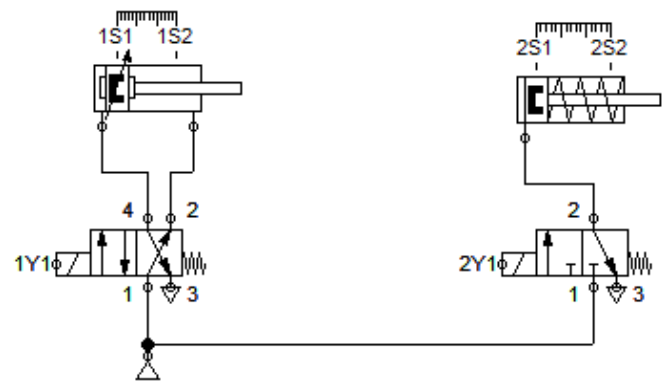

Fig. 23. Possible choice of actuators using $4 / 2$ and $3 / 2$ valves.

Mainly in electro-hydraulic circuits, with 3 positions valves, the spring acts to the central position, in which the actuator does not extend or retract. For these cases, the choice of spring returned valves is not possible, so the design should be done considering double solenoid 
valves.

Therefore, the final solution of the electric circuit by the optimized sequential method is given as in Fig. 24.

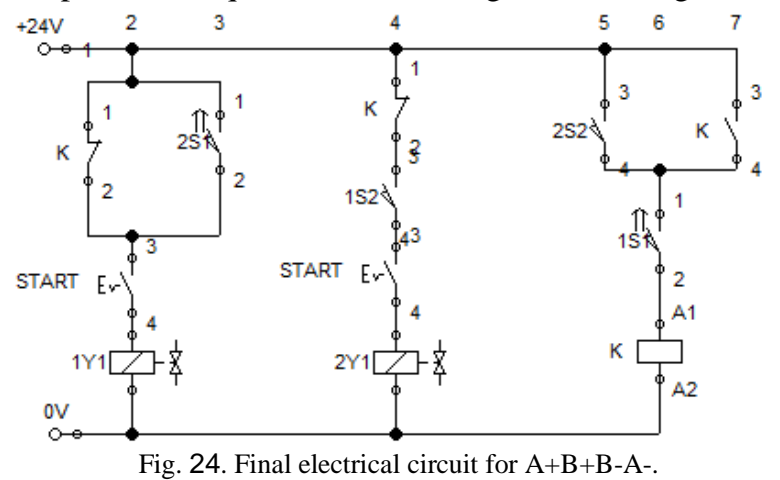

\subsection{Application with PLC}

The electric circuit obtained by the Optimized Sequential Method can be implemented using relays (Fig. 24) as well as through a PLC.

The LADDER code to be programmed in the PLC is obtained directly from the electrical circuit by simply setting the sensors and button as input signals, solenoids as output signals and relays as PLC internal Boolean variables, as shown in Table 1. The choice of names are made to represent the components in the LADDER code, but in general, the LADDER code does not support variable names beginning with numbers. Once the appropriate choices have been made, the LADDER code is then obtained according to Fig. 25.

Table 1. Ladder code variables: A+ B+ B- A-.

\begin{tabular}{c|c|c}
\hline COMPONENT & PLC I/O & $\begin{array}{c}\text { NAME OF } \\
\text { VARIABLE IN } \\
\text { LADDER CODE }\end{array}$ \\
\hline 1S1 & Input 1 & S11 \\
\hline 1S2 & Input 2 & S12 \\
\hline 2S1 & Input 3 & S21 \\
\hline 2S2 & Input 4 & S22 \\
\hline START BUTTON & Input 5 & START \\
\hline 1Y1 & Output 1 & Y11 \\
\hline 2Y1 & Output 2 & Y21 \\
\hline & $\begin{array}{c}\text { None } \\
\text { (Internal } \\
\text { variable). }\end{array}$ & K1
\end{tabular}

\subsection{Comparison with literature}

The sequence A+ B+ B- A- is also discussed by Santos and da Silva (2015), presenting the LADDER codes from three different methods, GRAFCET, Cascade and Karnaugh maps, as presented in Fig. 26.

The solution presented in Fig. 25 obtained by the proposed method is much more simpler if compared with Karnaugh maps, and more optimized in number of components (number of required relays, spring returned valves and actuators), connections and cost necessary to implement it if compared with the three mentioned methods.

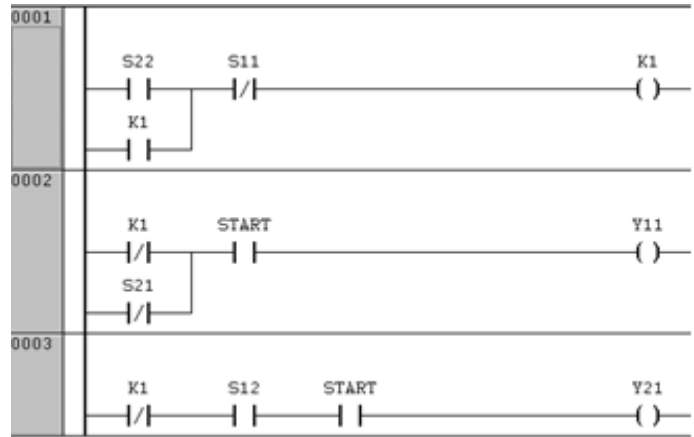

Fig. 25. LADDER code for A+B+B-A- from Optimized Sequential Method.

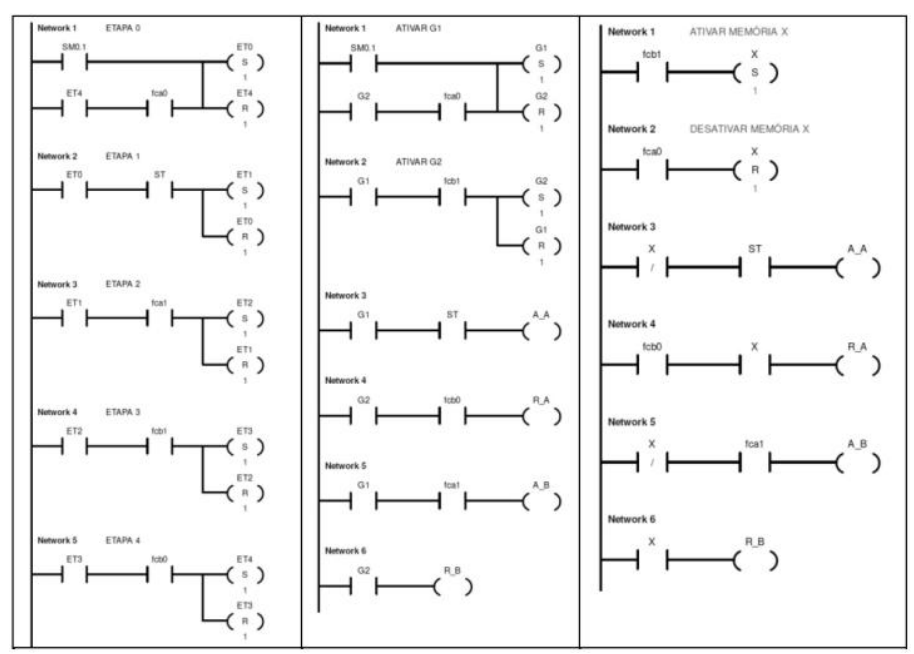

Fig. 26. LADDER codes from three different methods, GRAFCET,

Cascade and Karnaugh maps for sequence A + B + B- A- according to Santos and da Silva (2015).

The proposed method results in the LADDER code directly from the final electrical circuit, which does not require any additional computations. The ladder code is clear, it means that the technical team responsible for implementation and maintenance of the circuit can verify the code and also improve it without to ready pages of complex codes.

Another advantage is that the Optimized Sequential Method can be applied to more complex sequences, as follows.

\section{CIRCUIT WITH REPETITION AND SIMULTANEOUS MOVEMENTS}

To exemplify the application of the method in circuits with repetition and simultaneous movement, the solution of the sequence $\mathrm{A}+\mathrm{B}+\mathrm{A}-(\mathrm{A}+\mathrm{B}-) \mathrm{A}-$ is proposed, in which the parentheses indicate that the extend movement of $\mathrm{A}$ and retract of $\mathrm{B}$ occur simultaneously.

By dividing the sequence into the half by a vertical trace, an indirect command sequence is identified,

$\mathrm{A}+\mathrm{B}+\mathrm{A}-\mid(\mathrm{A}+\mathrm{B}-) \mathrm{A}-$.

Then the sequence is divided into sectors, according to Step 2 of Section 2.1, which results in:
(A
A-

I

A-
II


Since the sequence results in 4 sectors, 3 relays are required to carry out the command, as shown in Fig. 27.

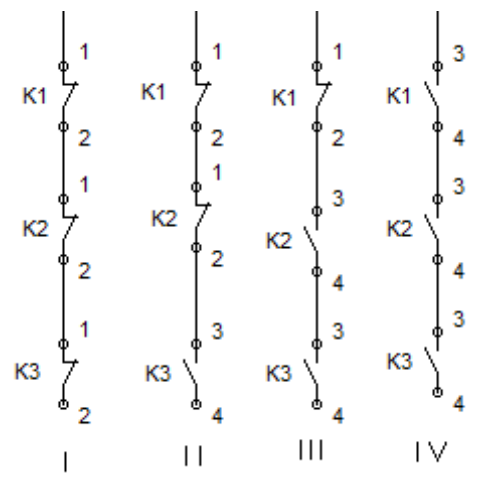

Fig. 27. An electric cascade of 4 sectors using 3 relays.

Then, the diagrams are made, according to Fig. 28, Fig. 29, Fig. 30 and Fig. 31.

From the diagrams, it is possible to obtain the equations of the commands using the AND/OR logic, according to the rules from Section 2.3.

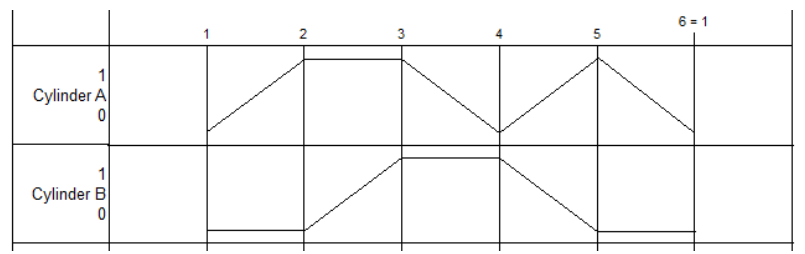

Fig. 28. Diagram of actuators of the sequence A+ B+ A- (A+B-) A-

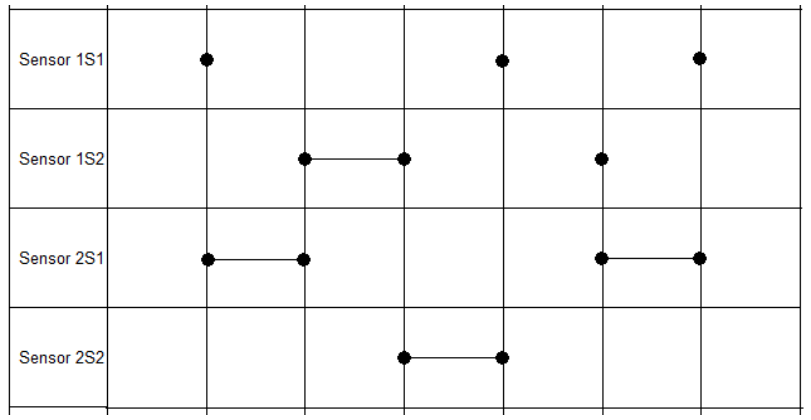

Fig. 29. Diagram of sensors of the sequence A+B+A- (A+B-) A-.

For the $A+$, an initial analysis can be done by (4).

$$
A+: 1 \mathrm{~S} 1 * \mathrm{~K} 1 \mathrm{NC} * \mathrm{~K} 2 \mathrm{NC} * \mathrm{~K} 3 \mathrm{NC}
$$

However, it is observed that identical response is obtained by (5).

$$
A+: 1 S 1
$$

Taking into account the comparison of the duration of $A+$, with the signal duration of $1 \mathrm{~S} 1$, it is observed that the duration of 1S1 is smaller than $A+$, which would lead to the use a solenoid to control $A+$.

The $A$ - can be expressed as (6).



Fig. 30. Diagram of the relays of the sequence A + B + A- (A+B-) A-.

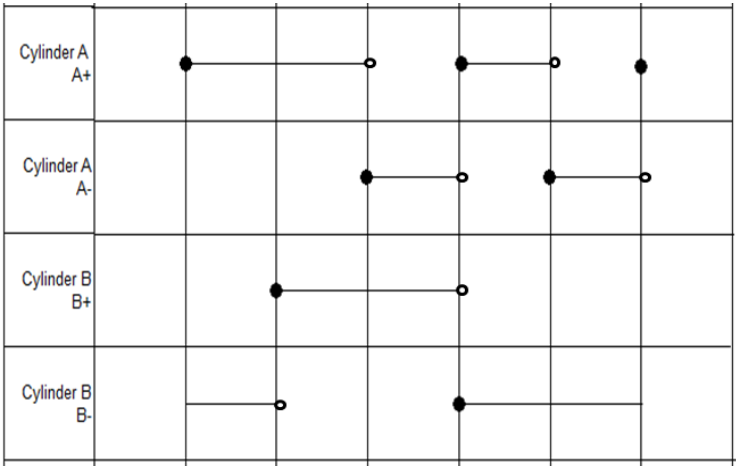

Fig. 31. Actuator control diagram of the sequence $A+B+A-(A+B-) A-$.

$$
A-: 2 \mathrm{~S} 2 . \mathrm{K} 1 \mathrm{NC} * \mathrm{~K} 2 \mathrm{NC} * \mathrm{~K} 3 \mathrm{NO}
$$

As $A$ - occurs twice during the sequence of motion, as shown in Fig. 32, it is necessary to evaluate the second stretch represented by (7).

$$
A-: 2 \mathrm{~S} 1 * \mathrm{~K} 1 \mathrm{NO} * \mathrm{~K} 2 \mathrm{NO} * \mathrm{~K} 3 \mathrm{NO}
$$

It should be noted, however, that in this case $A+$ and $B$ precede simultaneously the movement of the second stretch of $A$-, so the second movement of $A$ - can also be represented by (8).

$$
A-: \text { 1S1 } * \mathrm{~K} 1 \mathrm{NO} * \mathrm{~K} 2 \mathrm{NO} * \mathrm{~K} 3 \mathrm{NO}
$$

Equation (6) has a similar response if considered only the relay $\mathrm{K} 2 \mathrm{NC}$, instead of the association of all relays. Therefore, a simplification of relays is obtained in this case, resulting in (9).

$$
A-: 2 \mathrm{~S} 2 * \mathrm{~K} 2 \mathrm{NC}
$$

For the second movement of A-, we eliminate both the need for $\mathrm{K} 2 \mathrm{NO}$ and $\mathrm{K} 3 \mathrm{NO}$, in addition to the sensor, which could be $2 \mathrm{~S} 1$ or $1 \mathrm{~S} 2$, resulting in (10).

$A-:$ K $1 N O$ 




Fig. 32. The path-step diagram to obtain the equation of A-.

The final solution of A- must take into account both movements, (9) and (10), which can be done using an OR logic that is represented in (11).

$A-=(2 \mathrm{~S} 2 * \mathrm{~K} 2 \mathrm{NC})+\mathrm{K} 1 \mathrm{NO}$

Since the solution obtained for $A$ - has the same duration of the association of sensors and relays used, it is assumed that the valve used to control $A$ would be with one direction valve. In this case, as seen in subsection 2.3, one can obtain the logic of $A+$ from $A$-, which is given by (12).

$$
\begin{aligned}
& A+=\overline{A-}=\overline{(2 S 2 * K 2 N C)+K 1 N O} \\
& \mathrm{~A}+=(\overline{2 S 2}+K 2 N O) * K 1 N C
\end{aligned}
$$

Therefore, it is possible to replace the valve used to control $A$ to a spring returned valve, similar to Fig. 15 .

For actuator $B$, the diagrams are represented by Fig. 33 and Fig. 34.

According to the diagrams and applying the presented rules, it is verified that it is not possible to realize simplifications in the bidirectional valve, since the logic of activation of $B+$ and $B$-, already simplified, are given in (13).

$$
\begin{aligned}
& B+: 1 \mathrm{~S} 2 * \mathrm{~K} 2 \mathrm{NC} \\
& B-: \mathrm{K} 2 \mathrm{NO}
\end{aligned}
$$

Both cases have duration of actuation longer than the sensor and relay durations. In this way, the valve of $B$ must be bidirectional, similar to Fig. 16.

For the analysis of the seals, the rules presented in subsection 2.5 must be followed.

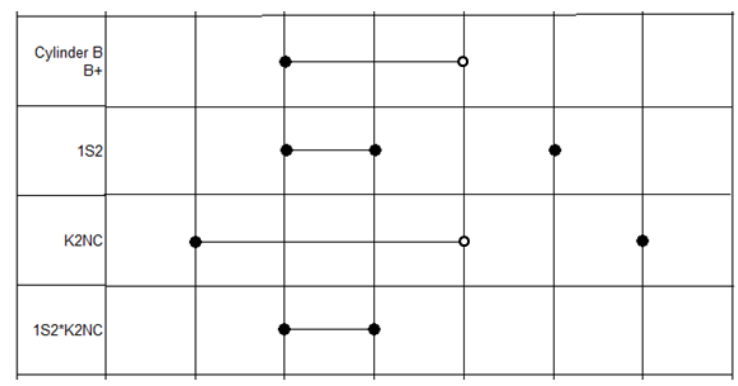

Fig. 33. The path-step diagram to obtain the $\mathrm{B}+$ equation.

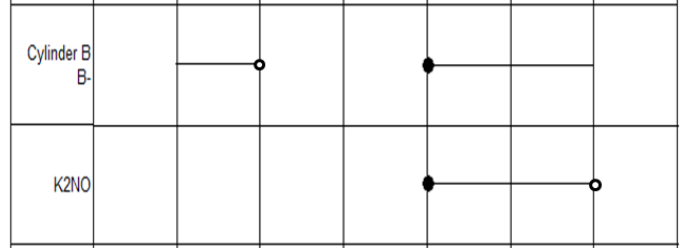

Fig. 34. The path-step diagram to obtain the B- equation

It should be noted that the control of actuators A and B did not require any contact of relay $\mathrm{K} 3$. In this way, a simplification resulting from the method is the elimination of the relay $\mathrm{K} 3$.

For the design of the $\mathrm{K} 1$ relay seal, the diagrams shown in Fig. 35 and Fig. 36 must be evaluated. From these diagrams it is possible to verify that $1 \mathrm{~S} 1 \mathrm{NC}$, has the open circles coincident with the K1NC signal, in other words, that the seal shut down is being performed correctly.

For the activation of the $\mathrm{K} 1$ seal, it is verified that the transition from $\mathrm{K} 1 \mathrm{NC}$ to $\mathrm{K} 1 \mathrm{NO}$ occurs after the simultaneous movement of $\mathrm{A}+$ (that trigger $1 \mathrm{~S} 2$ ) and $\mathrm{B}$ (that trigger $2 \mathrm{~S} 1$ ). In this way, the association of contacts responsible for triggering the $\mathrm{K} 1$ seal must be done considering $\overline{1 S 1} * 1 S 2$ or $\overline{1 S 1} * 2 S 1$. However, the $1 \mathrm{~S} 2$ sensor is already being used (in $\mathrm{B}+$ ), unlike the $2 \mathrm{~S} 1$ sensor, which again allows simplification, eliminating the need for the 2S1 sensor. Once the choice of $1 \mathrm{~S} 2$ has been made, the seal activation analysis is performed comparing $\mathrm{K} 1 \mathrm{NO}$ with $\overline{1 S 1} * 1 S 2$.

It is observed that $\overline{1 S 1} * 1 S 2$ results in a duration longer than $\mathrm{K} 1 \mathrm{NO}$, which leads to the need of associates $\overline{1 S 1} * 1 S 2$ with a contact from another sensor or even from another relay. When associating with the K2NO relay, one has that $\overline{1 S 1} * 1 S 2 * K 2 N O$ is smaller than $\mathrm{K} 1 \mathrm{NO}$ and start together, which results in a possible solution.

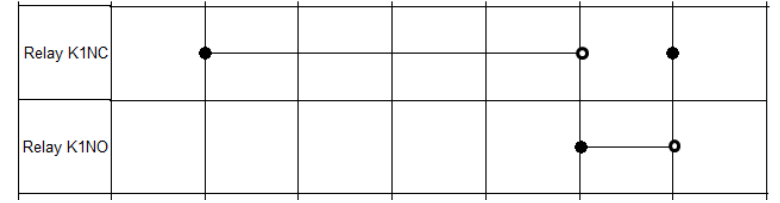

Fig. 35. Diagram of activation of the relay K1

For relay K2, the diagrams for the analysis of the seal are given by Fig. 37 and Fig. 38 .

From these diagrams it is possible to verify that $\overline{1 S 1}$, has an open circle in the 4th state, which does not coincide with $\mathrm{K} 2 \mathrm{NC}$, meaning that $\mathrm{K} 2 \mathrm{NC}$ is not performing correctly.

In this case, one should associate $\overline{1 S 1}$ with some contact that eliminates the open circle in the 4th state. One possible solution is to associate in parallel an normally open contact of the $2 \mathrm{~S} 2$ sensor. When comparing $\overline{1 S 1}+2 S 2$ with $\mathrm{K} 2 \mathrm{NC}$, it is found that the $\mathrm{K} 2$ seal shut down is done correctly, since all the open circles of the association coincide with $\mathrm{K} 2 \mathrm{NC}$. 


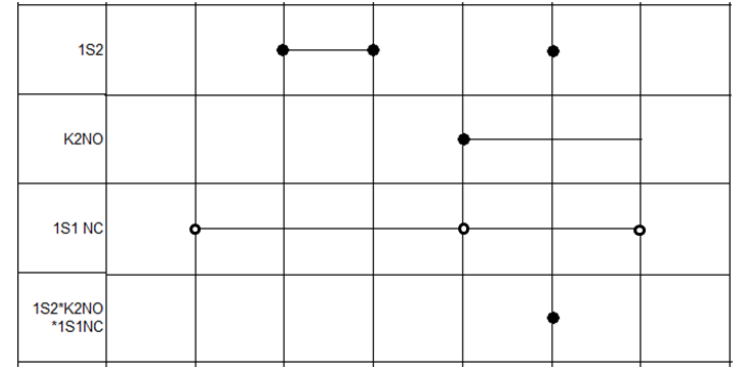

Fig. 36. Diagram of the seal of the relay K1.

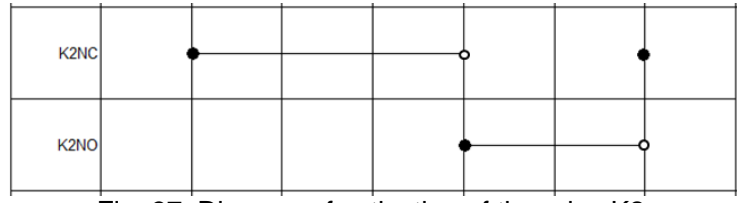

Fig. 37. Diagram of activation of the relay K2.

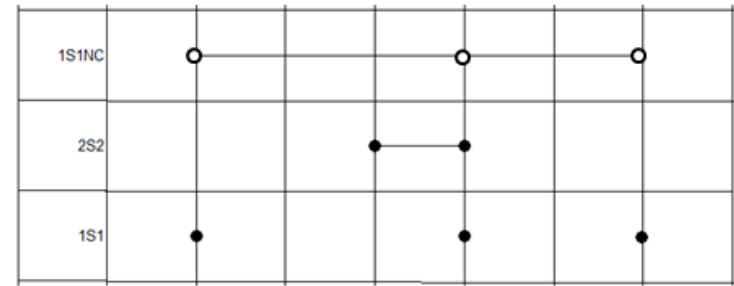

Fig. 38 Diagram of the seal of the relay K2.

The activation of the $\mathrm{K} 2$ seal is then done by comparing $1 S 1 *(\overline{1 S 1}+2 S 2)$, since $1 \mathrm{~S} 1$ (ON contact of seal) is in series with the previously associated contacts. Comparing $1 S 1 *(\overline{1 S 1}+2 S 2)$ with $\mathrm{K} 2 \mathrm{NO}$, it is verified that the signals start together and the duration of $\mathrm{K} 2 \mathrm{NO}$ is larger, which leads to a valid solution for the activation of the $\mathrm{K} 2$ seal.

In order to validate the proposed method, computational simulations (Waller and Werner, 2002) were made using Festo's Fluidsim software and also validated on an electro-pneumatic bench. The experimental tests were done using both electrical relays and through a PLC, using LADDER language.

The resulting electro-pneumatic circuit is shown in Fig. 39 and Fig. 40, and the mapping of the variables is given in Table 2.

The LADDER diagram is presented in Fig. 41. The circuits mounted on the electric stand and using the PLC are presented in Fig. 42 and Fig. 43. The tests proved the movement of the actuators as expected.

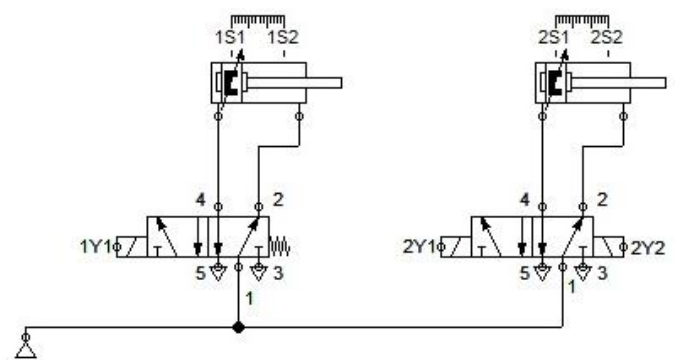

Fig. 39. Pneumatic connections of sequence $A+B+A-(A+B-) A-$.

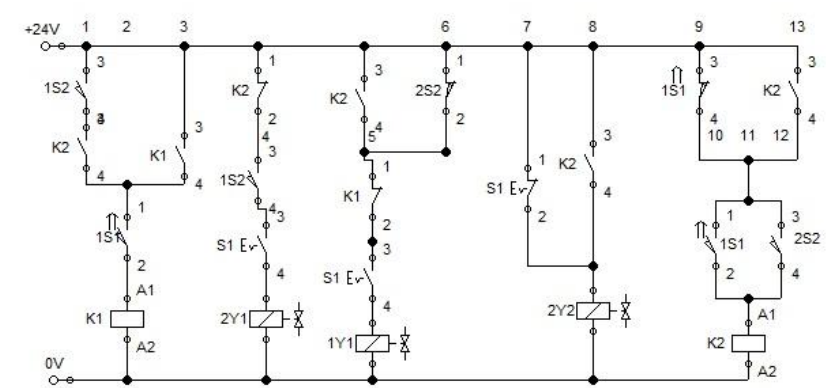

Fig. 40. Electrical connections of the sequence $A+B+A-(A+B-) A-$.

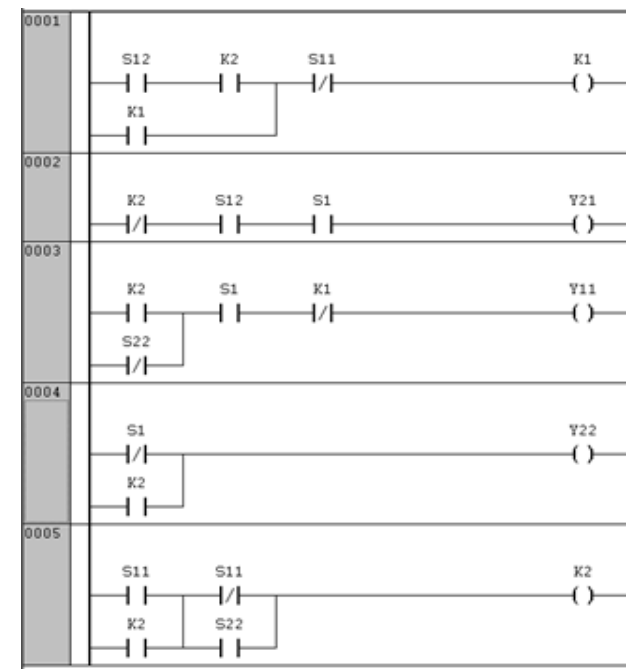

Fig. 41. LADDER code for sequence A+ B+ A- (A+B-) A-

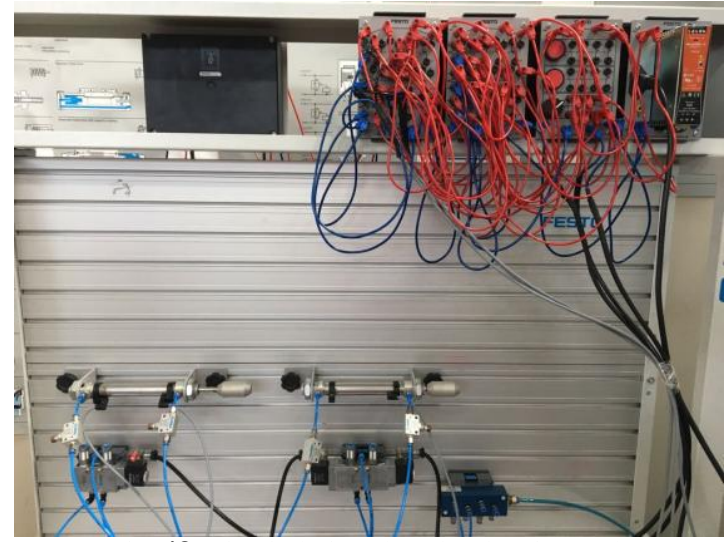

Fig. 42. Circuit designed on the electric stand

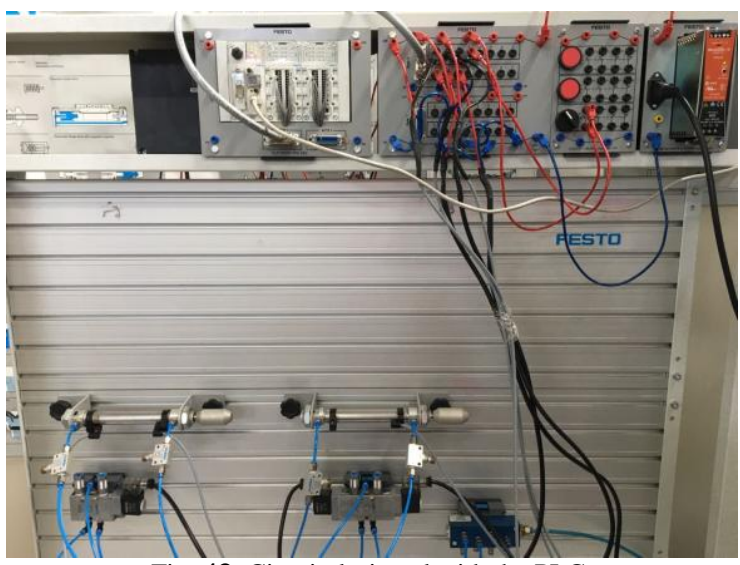

Fig. 43. Circuit designed with the PLC. 


\begin{tabular}{c|c|c}
\multicolumn{2}{c}{ Table 2. Ladder code variables for sequence A+ B+ A- (A+ B-) A-. } \\
\hline COMPONENT & $\begin{array}{c}\text { PLC } \\
\text { CHANNEL }\end{array}$ & $\begin{array}{c}\text { NAME OF VARIABLE } \\
\text { IN LADDER CODE }\end{array}$ \\
\hline 1S1 & Input 1 & S11 \\
\hline 1S2 & Input 2 & S12 \\
\hline 2S2 & Input 3 & S22 \\
\hline START BUTTON & Input 4 & Y11 \\
\hline 1Y1 & Output 1 & Y21 \\
\hline 2Y1 & Output 2 & K1 \\
\hline KY2 & Output 3 & $\begin{array}{c}\text { None } \\
\text { (Internal } \\
\text { variable). }\end{array}$ \\
\hline $\begin{array}{c}\text { None } \\
\text { (Internal } \\
\text { variable). }\end{array}$ & K2
\end{tabular}

\section{CONCLUSION}

The optimized sequential method proposes a solution for any actuators movement sequence for electropneumatic and electro-hydraulic circuits. With the use of the proposed method, it is possible to solve circuits that have simultaneous movement of actuators and circuits with repetition of the movement of an actuator. The method eliminates the need for the use of intuitive techniques, performed directly from the analysis of the diagrams of the actuators, sensors and relays.

When using the proposed method it is possible to simplify and optimize bidirectional solenoid valves for one directional ones. Another simplification resulting from the method is the elimination of relays and/or limit switches. Although the optimized sequential method produces an automation project with lower starting and maintenance cost, besides consuming less electricity.

The development of the new industrial automation project after the lockdown due COVID19, can or not use PLC, depending on products availability; therefore, the optimized sequential method also assists electric relay solution. However, PLC solution obtained by the method eliminates electronic components and relays, as they become internal PLC variables.

\section{V.REFERENCES}

1- Peter Rohner. Fluid Power Logic Circuit Design, Analysis, Design methods, and Worked Examples. Mecmillen press LTD., (1979).

2- José Novais. Método sequencial para automação eletropneumática. 3rd. Avenida de Berna (Lisbon), Portugal. Fundação Calouste Gulbenkian. 1997.

3- Mohamed S. Bayoumi. "Novel method for designing a sequential logic controller with Intermediate Stop of Actuators". International Journal of computer and information technology. Vol 3, pp. 643 - 650. May, 2014.

4- D. Waller, and H. Werner. Pneumatics, Workbook Basic Level. Festo Didactic GmbH \& Co., D-73770 Denkendorf, 2002.

5- S. Malayappan, K. A. Raj, S. S. Arunachalam, S V. D. Ramalinga. "Design of a sequential control circuit for an industrial robot using cascading method".
Proceeding of the international conference on manmachine systems. pp. 3B3-1 - 3B3-5. October, 2009.

6- T. Jagadeesha. Pneumatics: concepts, design and applications. 1st ed. Hyderabad 500029 (Telangana), India. Universities Press Private Limited. 2014.

7- Nelson G. Bonacorso, Valdir Noll. Automação eletropneumática. 9rd. Rua São Gil 159 (São Paulo), Brazil. Editora Eriça Ltda. 1997.

8- S. Ganesh, S.K. Gurunathan. "Hybrid intelligent systems for pneumatic sequential circuit design: Hybridizing genetic algorithms and rule-based logic programming". pp.241-246. $2011 . \quad$ DOI: 10.1109/HIS.2011.6122112.

9- S. Ganesh, S. K. Gurunathan. "Evolutionary algorithms for programming pneumatic sequential circuit controllers". International conference on flexible automation and intelligent manufacturing. pp. 1726 1734. June, 2017.

10- N. Mutyam, T.S.S.S Saykumar, B. Soppari. "Design of Sequential Electro-Pneumatic System". In: Satapathy S., Raju K., Molugaram K., Krishnaiah A., Tsihrintzis G. (eds) International Conference on Emerging Trends in Engineering (ICETE). Learning and Analytics in Intelligent Systems, vol 2. Springer, Cham, pp 610-615, July, 2019.

11- R.M.H. Cheng. "A Method of Analyzing the Logic Design of Pneumatic Sequential Circuits". In: Tobias S.A., Koenigsberger F. (eds) Proceedings of the Thirteenth International Machine Tool Design and Research Conference. Palgrave, London, 1973.

12- Santos, A. A., da Silva, A. F., "Electro Pneumatic Control versus Grafcet", Conference: International Congress on Education, Innovation and Learning Technologies At: Granada, Spain Volume: Vol. 1, pp. 6472, 2015.

13- MIT Emergency Ventilator (E-Vent) Project. Available at https://e-vent.mit.edu/. Accessed in 21/04/20.

14- Open Source Ventilator (OSV) - Irish project tackles global ventilator shortage. Available at https://www-rte-

ie.cdn.ampproject.org/c/s/www.rte.ie/amp/1124495/.

Accessed in 21/04/20.

15- Arno Bollmann. Fundamentos da Automação Industrial Pneutrônica - Projetos de commandos binários eletropneumáticos. ABHP (1996). 